\title{
NEIL3 may act as a potential prognostic biomarker for lung adenocarcinoma
}

\author{
Cui Zhao ${ }^{1+}$, Jian Liu ${ }^{3+}$, Haomiao Zhou ${ }^{1}$, Xin Qian ${ }^{1}$, Hui Sun ${ }^{2}$, Xuewen Chen ${ }^{4}$, Miaosen Zheng ${ }^{1}$, Tingting Bian², \\ Lei Liu ${ }^{2}$, Yifei Liu ${ }^{2^{*}+}$ and Jianguo Zhang ${ }^{2^{*}+}$ (D)
}

\begin{abstract}
Background: Lung adenocarcinoma (LUAD) is the leading cause of cancer-related death. This study aimed to develop and validate reliable prognostic biomarkers and signature.

Methods: Differentially expressed genes were identified based on three Gene Expression Omnibus (GEO) datasets. Based on 1052 samples' data from our cohort, GEO and The Cancer Genome Atlas, we explored the relationship of clinicopathological features and NEIL3 expression to determine clinical effect of NEIL3 in LUAD. Western blotting (22 pairs of tumor and normal tissues), Real-time quantitative PCR (19 pairs of tumor and normal tissues), and immunohistochemical analyses (406-tumor tissues subjected to microarray) were conducted. TIMER and ImmuCellAI analyzed relationship between NEIL3 expression and the abundance of tumor-infiltrating immune cells in LUAD. The coexpressed-gene prognostic signature was established based on the Cox regression analysis.

Results: This study identified 502 common differentially expressed genes and confirmed that NEIL3 was significantly overexpressed in LUAD samples $(P<0.001)$. Increased NEIL3 expression was related to advanced stage, larger tumor size and poor overall survival $(p<0.001)$ in three LUAD cohorts. The proportions of natural T regulatory cells and induced T regulatory cells increased in the high NEIL3 group, whereas those of B cells, Th17 cells and dendritic cells decreased. Gene set enrichment analysis indicated that NEIL3 may activate cell cycle progression and P53 signaling pathway, leading to poor outcomes. We identified nine prognosis-associated hub genes among 370 genes coexpressed with NEIL3. A 10-gene prognostic signature including NEIL3 and nine key co-expressed genes was constructed. Higher risk-score was correlated with more advanced stage, larger tumor size and worse outcome $(p<0.05)$. Finally, the signature was verified in test cohort (GSE50081) with superior diagnostic accuracy.
\end{abstract}

Conclusions: This study suggested that NEIL3 has the potential to be an immune-related therapeutic target and an independent predictor of LUAD prognosis. We also developed a prognostic signature for LUAD with a precise diagnostic accuracy.

Keywords: Bioinformatics, Lung adenocarcinoma, NEIL3, Real-time quantitative PCR, Immunohistochemistry, Prognostic signature

*Correspondence: ntdxliuyifei@sina.com; 13815212431@163.com

${ }^{\dagger}$ Cui Zhao, Jian Liu, Yifei Liu and Jianguo Zhang contributed equally to this work

${ }^{2}$ Department of Pathology, Affiliated Hospital of Nantong University, Nantong 226001, China

Full list of author information is available at the end of the article

\section{Background}

Lung cancer, accounting for almost one-quarter of all cancer deaths, has become more common than breast, brain, colorectal, and prostate cancers combined and is now the leading cause of cancer-related death globally [1]. Lung adenocarcinoma (LUAD) is the most frequent pathological subtype, accounting for nearly $45 \%$ of lung cancer cases [2]. The 5-year relative survival rate of

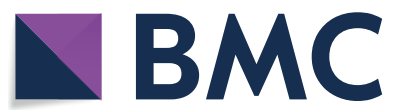

(c) The Author(s) 2021. This article is licensed under a Creative Commons Attribution 4.0 International License, which permits use, sharing, adaptation, distribution and reproduction in any medium or format, as long as you give appropriate credit to the original author(s) and the source, provide a link to the Creative Commons licence, and indicate if changes were made. The images or other third party material in this article are included in the article's Creative Commons licence, unless indicated otherwise in a credit line to the material. If material is not included in the article's Creative Commons licence and your intended use is not permitted by statutory regulation or exceeds the permitted use, you will need to obtain permission directly from the copyright holder. To view a copy of this licence, visit http://creativeco mmons.org/licenses/by/4.0/. The Creative Commons Public Domain Dedication waiver (http://creativecommons.org/publicdomain/ zero/1.0/) applies to the data made available in this article, unless otherwise stated in a credit line to the data. 
LUAD is only $5 \%$ because about $57 \%$ of patients are diagnosed with advanced stage and metastatic disease [3, 4]. Patients with LUAD have improved overall survival (OS) after surgery and radiotherapy when diagnosed sooner. With the development of immune checkpoint inhibitors (atezolizumab, pembrolizumab, nivolumab, etc.), the survival of LUAD patients has significantly improved; thus, such treatments have attracted considerable attention $[5,6]$. However, limited data are available regarding the relationship between biomarkers and immune responses. Therefore, exploring and identifying effective immunerelated biomarkers for LUAD to reduce the mortality rates and develop innovative targeted therapies remains crucial.

In the past ten years, researchers have conducted new studies and explored meaningful genes using bioinformatics techniques. Here we identified 502 commonly expressed genes from three LUAD datasets (GSE32863, GSE33532, and GSE43458). After an extensive literature review, we found that DNA endonuclease VIII-like 3 (NEIL3) is a very promising DNA glycosylase [7]. DNA glycosylase is one kind of enzymes to initiate the base excision repair (BER) pathway, recognize, and excise damaged bases from DNA [7-9]. NEIL3 has a glycolysis domain and apurinic/apyrimidinic lyase activity that excises damaged bases and generates a single-strand break [7, 9]. NEIL3 repairs telomere oxidative damage and protects telomere integrity during the $\mathrm{S}$ phase to enable accurate chromosome segregation in actively dividing cells [10]. Some findings based on a Neil3-/mice model indicated that the NEIL3 mutation was linked to impaired B cell function and severe autoimmunity [11]. NEIL3 promotes the proliferation of cardiac fibroblasts and neural progenitor cells in the brain and tends to be overexpressed in cells with a high proliferative capacity, such as cancer cells and those in the bone marrow $[8,12-14]$. The transcription of NEIL3 as a cell cycle dependent gene is regulated by BRG1 in breast cancer cells $[15,16]$. Moreover, NEIL3 is highly expressed in primary malignant melanomas, which may induce metastatic tumors [17]. Much evidence suggests that NEIL3 as a DNA repair gene is connected to tumorigenesis, therapy resistance, and poor prognosis in astrocytoma [18, 19]. Evidence has shown that NEIL3 participates in regulating the cell proliferation process. However, the association between NEIL3, cancer prognosis, and immune response in LUAD is unclear.

Here we analyzed the correlation between NEIL3 expression and the clinical characteristics, and the prognosis of LUAD patients based on the gene expression and clinicopathology data of three different cohorts [The Cancer Genome Atlas (TCGA), Gene Expression Omnibus (GEO), and our patient group]. We also investigated the relationship between NEIL3 expression and tumorinfiltrating immune cells using Immune Cell Abundance Identifier (ImmuCellAI) and Tumor Immune Estimation Resource (TIMER). To better understand the role of NEIL3 in LUAD development, we performed a gene set enrichment analysis (GSEA) and screened out 370 NEIL3 co-expressed genes. Importantly, nine hub genes were independent predictors for LUAD; thus, we attempted to construct a prognostic signature and validate its prognostic accuracy in other cohorts.

\section{Materials and methods}

\section{Human tissue samples}

Twenty-two pairs of frozen LUAD and adjacent noncancerous tissues obtained from the Affiliated Hospital of Nantong University between March 2018 and June 2019 were subjected to real-time quantitative polymerase chain reaction (RT-qPCR) and western blotting. Fresh tissues were frozen in liquid nitrogen and stored at $-80{ }^{\circ} \mathrm{C}$ until the experiment. LUAD tissue microarray and clinical data for 406 tumor samples and 50 normal lung tissue samples were derived from the Pathology Department of the Affiliated Hospital of Nantong University and used as our cohort. The inclusion criteria were as follows: (1) lobectomy and pneumonectomy with mediastinal lymph node dissection or sampling and (2) tumor diagnosed as invasive lung adenocarcinoma by postoperative pathology examination. The exclusion criteria were as follows: (1) no mediastinal lymph node dissection or sampling; (2) a history of other malignancies; (3) miss key clinical information such as age, sex, TNM stage, overall survival, distant and lymph node metastasis. Informed consent was obtained from all patients before the study. The ethical committee of Affiliated Hospital of Nantong University approved the study (number: 2018-L068), which was also conducted according to the Declaration of Helsinki.

\section{Data resources and preprocessing}

The GEO cohort included five gene expression datasets (http://www.ncbi.nlm.nih.gov/geo). GSE33532, GSE30219, and GSE43458 were used to screen out differentially expressed genes (DEGs) between LUAD and adjacent lung tissues by GEO2R $(\operatorname{logFC}>1$; adjusted $p<0.001)$, which included 40 LUAD tissues and 20 adjacent lung tissues, 85 LUAD tissues and 14 adjacent lung tissues and 80 LUAD tissues and 30 adjacent lung tissues, respectively. The GEO cohort also included GSE31210 (226 LUAD tissues and 20 adjacent lung tissues) and GSE50081 (127 LUAD tissues of stage I and II), which were used to explore the relationship between NEIL3 expression and the clinical outcomes of LUAD patients.

We utilized the gene expression profile and clinical information of the TCGA cohort (workflow type: 
HTSeq-FPKM; https://portal.gdc.cancer.gov/proje cts), and determined NEIL3 gene expression using $\mathrm{R}$ software (version: 3.5.3) and Strawberry Perl (version: 5.30.2.1). The TCGA cohort included 293 tumor samples and 54 normal lung tissues after the elimination of samples for which key clinical information was missing such as age, sex, tumor-node-metastasis stage (according to the 8th edition of the AJCC TNM staging system), overall survival, distant and lymph node metastasis. Meanwhile cases with a follow-up time of less than 90 days were deleted. Our work was performed in accordance with the TCGA publication requirements.

\section{Functional enrichment analyses}

GSEA, a powerful calculation software (http://softw are.broadinstitute.org/gsea/index.jsp) based on Gene Ontology (GO) and the Kyoto Gene and Genomic Encyclopedia (KEGG), was used to investigate the possible biological functions of NEIL3. The cohort of LUAD patients was divided into high and low NEIL3 expression groups by median values. The gene sets used in this work (c2.cp.kegg.v5.2.symbols.gmt) were downloaded from the Molecular Signatures Database (http://software.broad institute.org/gsea/msigdb/index.jsp) [20]. We performed functional enrichment of the NEIL3 and co-expressed genes using the Bohao Online Enrichment Tool (http:// enrich.shbio.com/) [21]. When a false discovery rate (FDR) and the nominal $p$ were less than 0.05 , the enrichment results were deemed statistically significant.

\section{Immune infiltrates analysis}

TIMER is a user-friendly web tool that is used to investigate the molecular characterization of tumor immune system interactions including six major analytic modules (https://cistrome.shinyapps.io/timer/). We evaluated the correlation between NEIL3 expression and the abundance of the six tumor-infiltrating immune subsets in LUAD: B cells, CD4+ T cells, neutrophils, CD8+ T cells, dendritic cells (DCs), and macrophages [22]. ImmuCellAI has a powerful ability for tumor-immune infiltration estimation, especially in the abundance of 18T-cell subsets (http://bioinfo.life.hust.edu.cn/ImmuCellAI\# !/) [23]. A gene set signature of TCGA LUAD patients was uploaded to the website. ImmuCellAI predicted the abundance of 24 immune cells in the sample including $18 \mathrm{~T}$-cell subsets. We measured the immune response of 24 immune cells to evaluate their association with NEIL3 expression in LUAD and performed "vioplot" package to visualize the data. At values of $p<0.05$, the results were considered statistically significant.
Predictive signature construction and risk score calculation The Cox proportional hazards model is often applied to survival analyses. We selected out nine prognostic signatures from among the 10 hub genes via "Survival" package and Cox regression analysis. We also performed a multivariate Cox regression analysis including NEIL3 and nine prognostic signatures, and constructed a 10-gene prognostic model to evaluate individual survival risk as follows: risk score $=0.00043 \times N E I L 3$ expression level $+(-0.00907) \times C C N B 2$ expression level $+0.00357 \times C D K 1 \quad$ expression level $+(-0.04486) \times C D C 45$ expression level $+0.05000 \times B U B 1 B$ expression level $+(-0.04195) \times B U B 1 \quad$ expression level $+(-0.0003) \times K I F 23$ expression level $+0.047506 \times C C N A 2$ expression level $+(-0.00037) \times U B E 2 C \quad$ expression level $+0.1721 \times N C A P G$ expression level. The optimal cutoffs and related specificity and sensitivity from receiver operating characteristic (ROC) curves were determined using a conventional method. Values of $p<0.05$ were considered statistically significant.

\section{Quantitative real time polymerase chain reaction}

All reactions were performed on a Mastercycler ep realplex (Eppendorf, Hamburg, Germany). The reaction conditions were $95{ }^{\circ} \mathrm{C}$ for $5 \mathrm{~s}, 60{ }^{\circ} \mathrm{C}$ for $30 \mathrm{~s}$, and $72{ }^{\circ} \mathrm{C}$ for $1 \mathrm{~min}$ for a total of 40 cycles. GAPDH was used as the internal control. The RT-qPCR primer sequences were as follows: NEIL3 forward primer, 5'-TACAGGTGCCGT AAAGCAGG-3' and reverse primer, 5'-GCGAGGGCT GTCAGGATTTA-3'; GAPDH forward primer, 5'-GAT CATCAGCAATGCCTCCTG-3'; and reverse primer, 5'-GAGTCCTTCCACGATACCAAAG-3'.

\section{Western blotting assay}

Fresh tissues and protein lysis buffer (including protease inhibitors) were added in homogenizers and grinded fully for extracting proteins. Protein was measured using the bicinchoninic acid method, and separated on a $12 \%$ sodium dodecyl sulfate polyacrylamide gel via electrophoresis (cat. \#XP00100BOX; Thermo, USA). The protein was then transferred onto polyvinyl difluoride membranes (cat. \#88518; Thermo), and the membranes were blocked with $5 \%$ skim milk in Tris-buffered saline with Tween-20. After the membranes were incubated with rabbit anti-NEIL3 (1:2000 dilution; cat. \#PA551022; Thermo) or $\beta$-actin overnight at $4{ }^{\circ} \mathrm{C}(1: 10000$ dilution; cat. \#AM4302; Thermo) and then incubated with anti-rabbit secondary antibodies (1:10,000 dilution; cat. \#A32733; Thermo) or anti-mouse secondary antibodies (1:2000 dilution; cat. \#A-10654; Thermo) for $1 \mathrm{~h}$. The enhanced chemiluminescence technique was used to develop the signals. The density of the protein bands was quantified by ImageJ software (National Institutes of 
Health, Bethesda, MD) and normalized to $\beta$-actin. Relative protein levels were calculated as the density ratios of interest protein to $\beta$-actin.

\section{Immunohistochemistry assay}

The slides of LUAD tissues were stained with rabbit antiNEIL3 (1:400 dilution; cat. \#PA5-51022; Thermo), followed by anti-rabbit secondary antibody (1:2000 dilution; cat. \#ab205718; Abcam) and diaminobenzidine treatment. Immunohistochemistry score (IHC-score) = staining intensity score $\times$ staining area ratio score of positive cells. The staining intensity was scored as 0 for negative, 1 for weakly positive, 2 for medium positive, and 3 for strongly positive. The positive area ratio was scored as $0(0 \%), 1(1-25 \%), 2(26-50 \%), 3(51-75 \%)$, or $4(76-$ 100\%) [24]. When the IHC-score was less than 6 , the case was classified into the low expression group; otherwise, the case was classified into the high expression group. The staining results were independently scored by two pathologists.

\section{Statistical analysis}

All statistical analyses were performed using R (v.3.5.3) and SPSS version 15.0 (SPSS Inc., Chicago, IL, USA). 22 pairs of tumor and normal tissues were used in Western blotting, which statistical power is 0.8291 . 19 pairs of tumor and normal tissues were used in PCR, which statistical power is 0.8130 . Statistical power calculations are performed using STATA v.15.0. To evaluate the correlation between NEIL3 expression and the other variables (sex, age, TNM stage, tumor size, distant metastasis, and OS), we performed Spearman correlation test, Chisquare Tests and Wilcoxon/Kruskal-Wallis test. We stratified the cohort into patients with a high or low median NEIL3 expression value or median risk score as the cut-off value. Multivariate Cox regression analysis was conducted to identify independent prognostic factors. Values of $p<0.05$ were considered as statistically significant.

\section{Results}

\section{NEIL3 overexpression in LUAD}

We thoroughly screened the LUAD data in the GEO database and selected three miRNA-sequencing datasets: GSE30219, GSE43458, and GSE33532. We identified 502 common DEGs among them (Fig. 1a), including 126 up-regulated DEGs in cancer tissues (Fig. 1b). After an extensive literature review, we found that NEIL3 is a very promising DNA repair gene. TIMER data showed that NEIL3 expression increased in 19 kinds of tumor tissues compared to adjacent normal tissues, especially in LUAD (Fig. 1c).

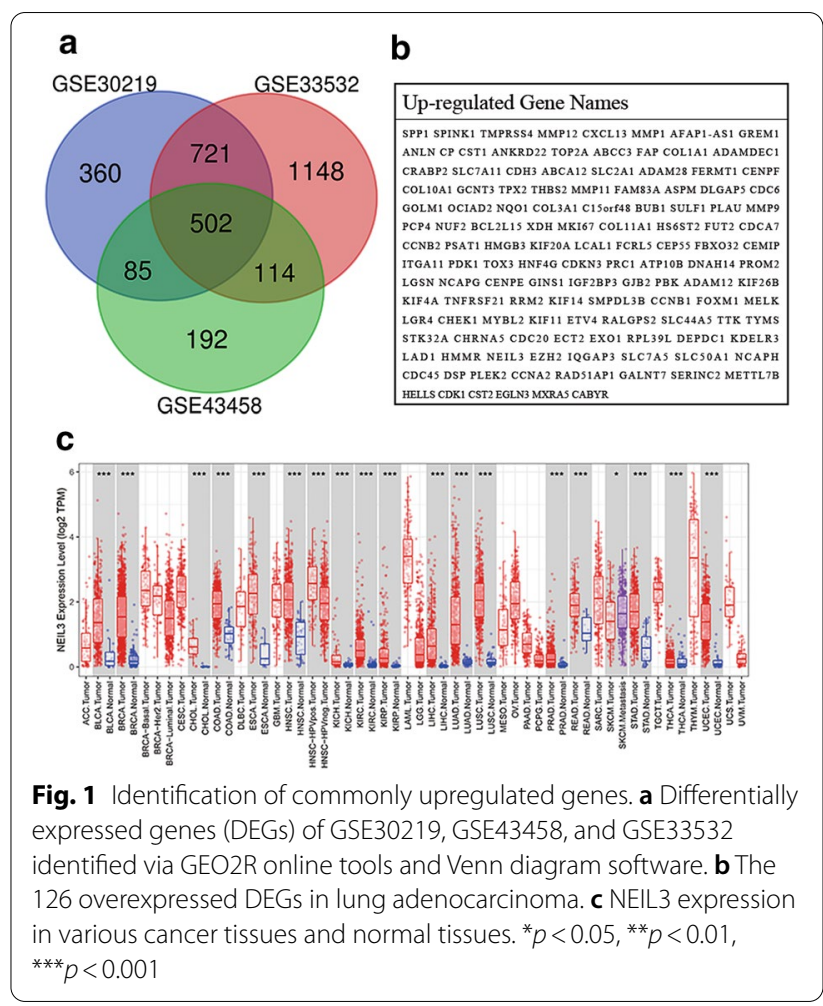

Analysis of the NEIL3 gene expression data in the TCGA and GSE31210 cohorts confirmed that NEIL3 is overexpressed in LUAD tissue (Fig. 2a, b). Therefore, RT-qPCR and western blotting were verified that the NEIL3 protein and mRNA levels were up-regulated in 22 LUAD tissues compared with the matched normal tissues (Fig. 2c, d). NEIL3 staining was positive in LUAD tissues but negative in normal lung tissue after immunohistochemistry staining (Fig. 2e). The results consistently showed that NEIL3 was obviously overexpressed in LUAD compared with the matched normal tissues $(p<0.001)$ (Fig. 2).

\section{Correlation between NEIL3 expression and clinicopathological characteristics of LUAD patients} The characteristics of our 406 LUAD cohort subjects and 293 TCGA LUAD cohort subjects are summarized in Tables 1 and 2. All samples were divided into low and high expression groups by IHC-score and median expression level. The results revealed that NEIL3 overexpression was closely associated with advanced TNM stage $(p<0.05$; Fig. 3a) and larger tumor size $(p<0.05$; Fig. 3b, $c)$. No significant correlation was noted between NEIL3 expression and other clinicopathological characteristics. Previous research has proved that NEIL3 could protect telomere integrity during the $S$ phase and accurately segregate chromosomes in actively dividing cells [10], which 

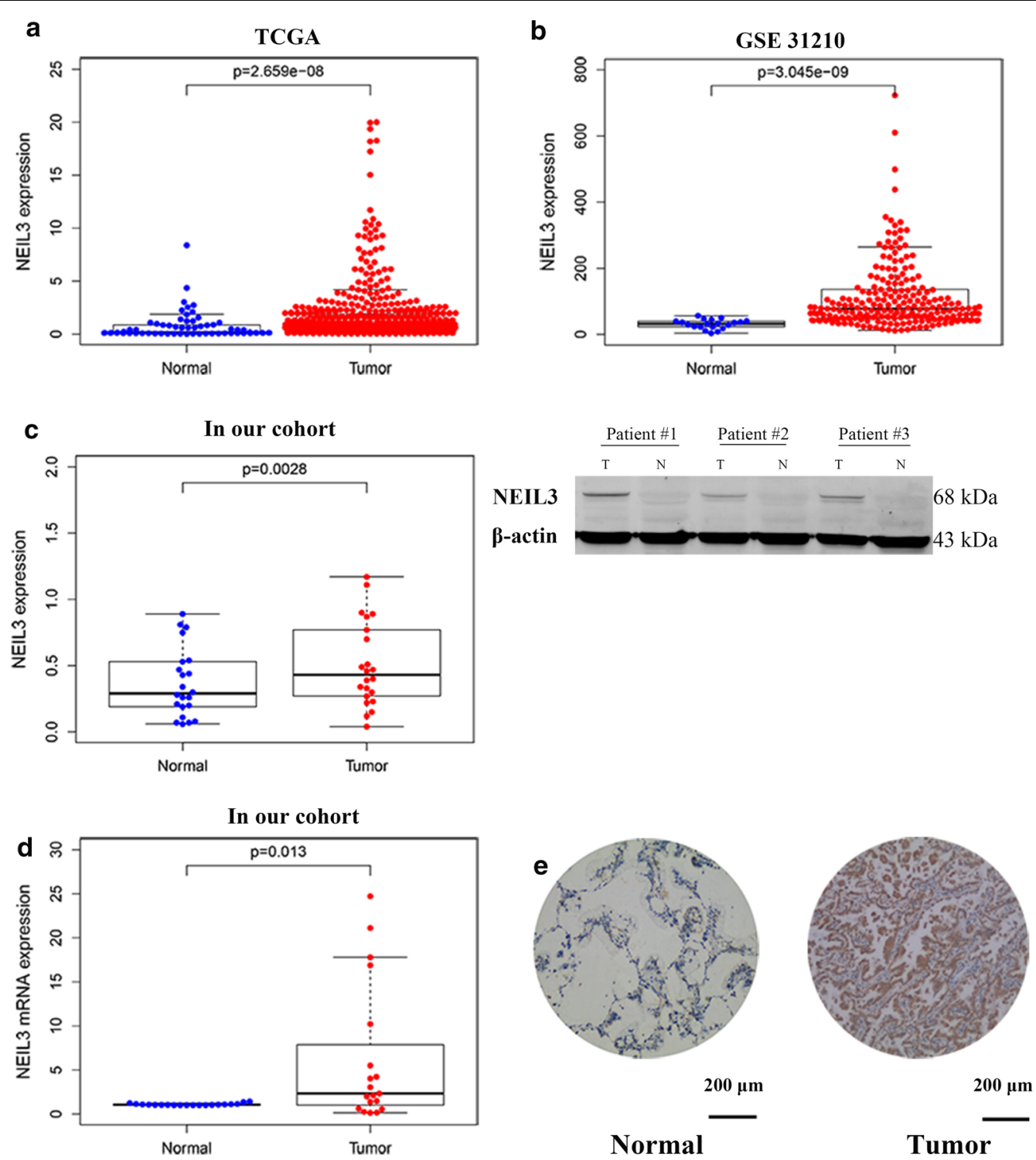

Fig. 2 NEIL3 was overexpressed in LUAD. a, b The NEIL3 expression in TCGA LUAD cohort and GSE31210 cohort. c NEIL3 protein level in 22 pairs of LUAD tissues and their matched normal tissues were determined by western blotting. $\mathbf{d}$ NEIL3 mRNA levels were determined by quantitative real-time polymerase chain reaction (qRT-PCR). e Immunohistochemical staining for NEIL3 in normal lung tissue and lung adenocarcinoma tissues (original magnification $\times 100$ ). N normal tissue, $T$ tumor tissue

may explain the advanced T classification in NEIL3-overexpressing patients.

\section{NEIL3 overexpression predicts poor prognosis in LUAD patients}

This study included all follow-up survival information of the GSE50081, GSE31210, TCGA datasets and our cohort. As is shown in Fig. 3d-g, Kaplan-Meier curve and log-rank test analyses indicated that up-regulated NEIL3 expression significantly reduced the OS and RFS of LUAD patients $(p<0.05)$. All risk factors and NEIL3 expression levels were subjected in the univariate Cox regression analysis (Table 3); of them, these were associated with OS: TNM stage, tumor size, lymph node metastasis, and NEIL3 expression $(p<0.001)$. Moreover, the multivariate Cox analysis result showed that increased NEIL3 expression [hazard ratio $(\mathrm{HR})=1.638$, 95\% confidence interval (CI) $1.338-2.005 ; p<0.001$ ] and advanced TNM stage (HR $=1.305,95 \%$ CI $1.021-$ $1.669 ; p<0.05)$ were independent predictors of poor prognosis (Table 3 and Fig. 3 h). 
Table 1 Relationship between NEIL3 expression and clinicopathology in the TCGA LUAD cohort

\begin{tabular}{|c|c|c|c|c|c|}
\hline \multirow[t]{2}{*}{ Characteristics } & \multirow[t]{2}{*}{$\mathrm{n}$} & \multicolumn{2}{|l|}{ NEIL3 } & \multirow[t]{2}{*}{$x^{2}$} & \multirow[t]{2}{*}{$P$ value } \\
\hline & & Low expression & High expression & & \\
\hline Total & 293 & 147 & 146 & & \\
\hline Age & & & & 0.206 & 0.65 \\
\hline$\leq 60$ & 98 & 51 (52.05) & $47(47.95)$ & & \\
\hline$>60$ & 195 & $96(49.24)$ & 99 (50.76) & & \\
\hline Gender & & & & 2.13 & 0.144 \\
\hline Male & 138 & $63(45.65)$ & 75 (54.35) & & \\
\hline Female & 155 & $84(54.19)$ & $71(45.81)$ & & \\
\hline TNM stage & & & & 7.689 & $0.004^{*}$ \\
\hline | and || & 223 & $122(54.70)$ & $101(45.30)$ & & \\
\hline III and IV & 70 & $25(35.70)$ & $45(64.30)$ & & \\
\hline Tumor stage & & & & 7.201 & $0.027^{*}$ \\
\hline I & 88 & $54(61.40)$ & $34(38.60)$ & & \\
\hline$\|$ & 169 & $80(47.30)$ & $89(52.70)$ & & \\
\hline III and IV & 37 & $14(37.80)$ & $23(62.20)$ & & \\
\hline Metastasis stage & & & & 2.809 & 0.094 \\
\hline Negative & 274 & $141(51.50)$ & $133(48.50)$ & & \\
\hline Positive & 19 & $6(31.60)$ & $13(68.40)$ & & \\
\hline Node stage & & & & 2.259 & 0.133 \\
\hline Negative & 187 & $100(53.50)$ & $87(46.50)$ & & \\
\hline Positive & 106 & $47(44.30)$ & $59(55.70)$ & & \\
\hline
\end{tabular}

*Statistically significant

NEIL3 expression is associated with immune cell infiltration in LUAD

Patients with the same histological type of cancer may have different degree of immune infiltration cells that lead to diverse clinical outcomes [22, 25]. The fact that an increased number of tumor-infiltrating lymphocytes in primary tumor tissue relates to good prognosis has been reported in several cancers, including LUAD [26]. The TIMER result showed that NEIL3 expression had a significant negative correlation with the infiltration of B cells, CD4+ T cells, and DCs $(p<0.05$; Fig. $4 \mathrm{a})$. The TIMER "Survival" module showed that high infiltrating levels of B cells benefit OS in contrast to NEIL3 expression $(p<0.05$; Fig. $4 \mathrm{~b})$. We speculated that NEIL3 overexpression could affect $\mathrm{OS}$ by regulating the degree of B-cell infiltration in LUAD.

The ImmuCellAI analysis showed that the frequencies of natural $\mathrm{T}$ regulatory ( $\mathrm{n}$ Treg), induced $\mathrm{T}$ regulatory (iTreg), Th1 cells, exhausted T cells, NK cells, effector memory and Gamma delta $\mathrm{T}$ cells exhibited a positive correlation with NEIL3 expression $(p<0.001)$, whereas the proportion of CD4 naïve cells, Tregulatory1 ( $\operatorname{Tr} 1)$, Th17, follicular helper T cell (Tfh), NK T, DC, and CD4 T cells exhibited a negative correlation $(p<0.001)$ (Fig. $4 c)$. This funding suggested that NEIL3 has a regulatory
Table 2 Relationship between NEIL3 expression and clinicopathology in our LUAD cohort

\begin{tabular}{|c|c|c|c|c|c|}
\hline \multirow[t]{2}{*}{ Characteristics } & \multirow[t]{2}{*}{$n$} & \multicolumn{2}{|l|}{ NEIL3 } & \multirow[t]{2}{*}{$x^{2}$} & \multirow[t]{2}{*}{$P$ value } \\
\hline & & $\begin{array}{l}\text { Low } \\
\text { expression }\end{array}$ & $\begin{array}{l}\text { High } \\
\text { expression }\end{array}$ & & \\
\hline Total & 406 & 228 & 178 & & \\
\hline Age & & & & 1.082 & 0.298 \\
\hline$\leq 60$ & 146 & $77(52.74)$ & $69(47.26)$ & & \\
\hline$>60$ & 260 & $151(58.08)$ & 109 (41.92) & & \\
\hline Gender & & & & 2.814 & 0.093 \\
\hline Male & 249 & $148(59.44)$ & $101(40.56)$ & & \\
\hline Female & 157 & $80(50.96)$ & $77(49.04)$ & & \\
\hline TNM stage & & & & 1.81 & 0.178 \\
\hline | and || & 312 & $169(54.17)$ & $143(45.83)$ & & \\
\hline III and IV & 94 & $59(62.77)$ & $35(37.23)$ & & \\
\hline Tumor stage & & & & 14.648 & $0.002^{*}$ \\
\hline I & 203 & $124(61.08)$ & $79(38.92)$ & & \\
\hline$\|$ & 145 & $81(55.86)$ & $64(44.14)$ & & \\
\hline III & 32 & $8(25.00)$ & $24(75.00)$ & & \\
\hline IV & 26 & 15 (57.69) & $11(42.31)$ & & \\
\hline Metastasis stage & & & & 0.001 & 0.971 \\
\hline Negative & 397 & $223(56.17)$ & $174(43.83)$ & & \\
\hline Positive & 9 & $5(55.56)$ & $4(44.44)$ & & \\
\hline Node stage & & & & 0.88 & 0.348 \\
\hline Negative & 222 & $120(54.05)$ & $102(45.94)$ & & \\
\hline Positive & 184 & $108(58.70)$ & $76(41.30)$ & & \\
\hline
\end{tabular}

*Statistically significant

effect on the formation of the LUAD immune microenvironment, especially on T-cell subsets, NK cells, and DCs. Figure $4 \mathrm{~d}$ shows the correlation between different types of immune cell subsets. Exhausted T cells had the strongest positive correlation with Cytotoxic $\mathrm{T}$ lymphocyte (Pearson correlation $=0.73$ ), while Cytotoxic $\mathrm{T}$ lymphocyte had the strongest negative correlation with CD4 naïve cells (Pearson correlation $=-0.66$ ). Taken together, these findings indicate that NEIL3 plays a key role in the regulation of immune-infiltrating cells in LUAD.

\section{KEGG and GO enrichment analysis of NEIL3 and co-expressed genes in LUAD}

According to FDR $<0.050$ and normalized enrichment score, the GSEA analysis revealed that the pathways of the cell cycle, nucleotide excision repair, DNA replication, mismatch repair, and the P53 signaling pathway were enriched in the high NEIL3 expression phenotype (Fig. 5a). The asthma and aldosterone-regulated sodium reabsorption pathways were enriched in the low NEIL3 expression cohort (Fig. 5a). It is worth noting that the cell cycle pathway, playing a crucial part in tumorigenesis and development, is associated with NEIL3 expression. 

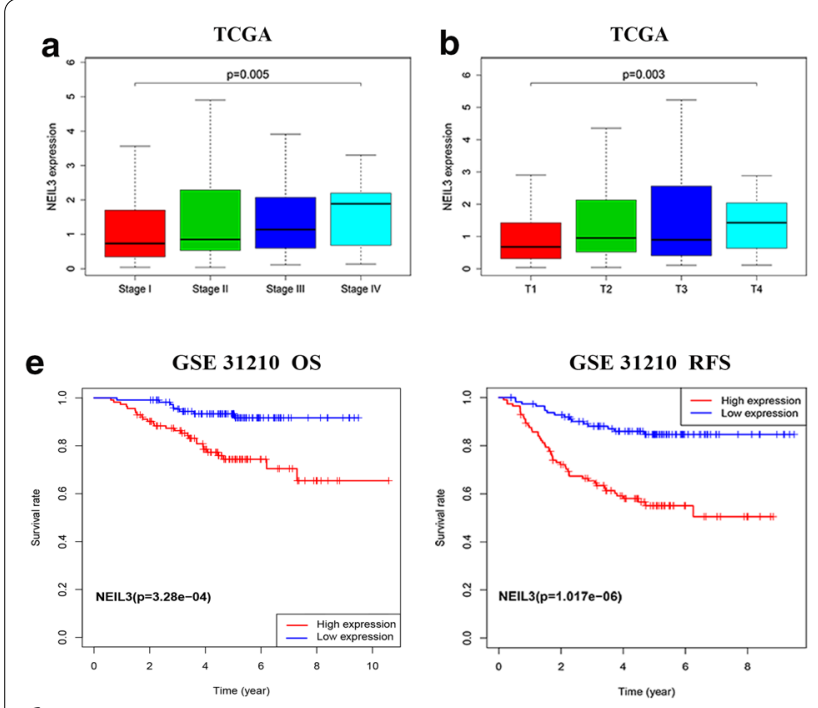

f

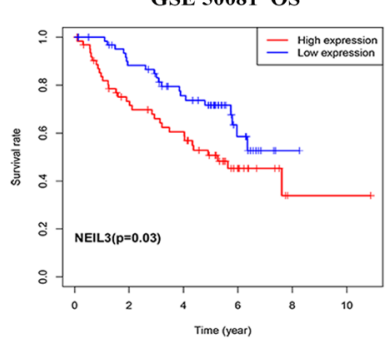

g

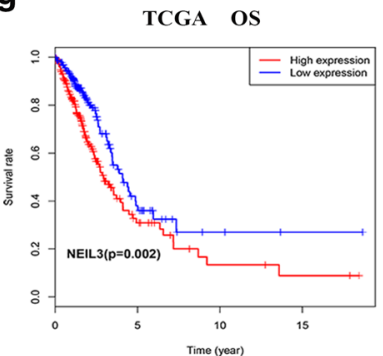

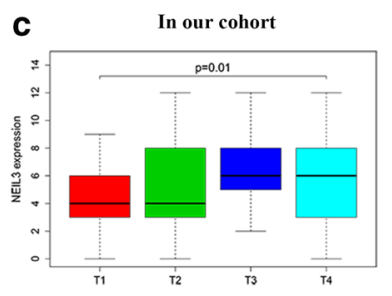
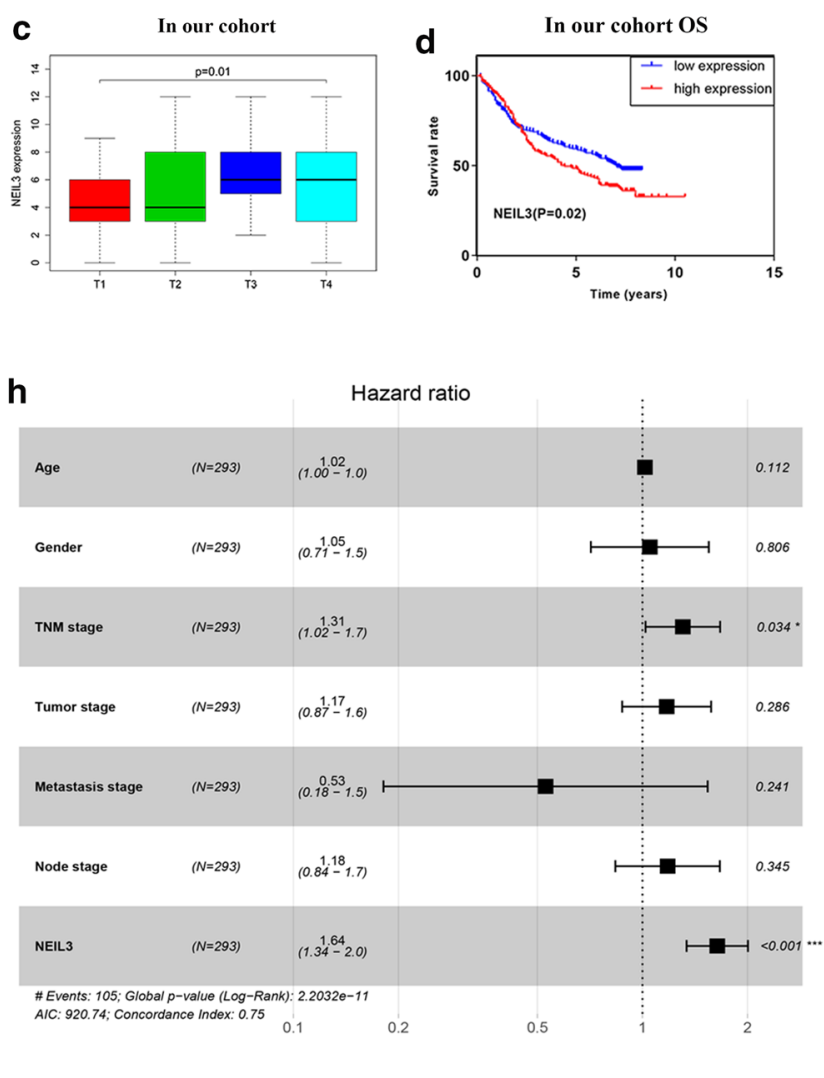

h

Fig. 3 Association with NEL3 expression, clinicopathological characteristics, overall survival (OS), and relapse-free survival (RFS). a-c Increased NEIL3 expression was significantly associated with advanced TNM stage and larger tumor size. $\mathbf{d}-\mathbf{g}$ The Kaplan-Meier plotters based on four datasets showed LUAD patients with high NEIL3 expression have poor OS and RFS. $\mathbf{h}$ The multivariate analyses proved the independent predictive ability of TNM stage and NEIL3 expression for LUAD overall survival

Table 3 Univariate and multivariate analyses of factors associated with overall survival in LUADs using Cox regression

\begin{tabular}{|c|c|c|c|c|}
\hline \multirow[t]{2}{*}{ Variable } & \multicolumn{2}{|l|}{ Univariate } & \multicolumn{2}{|l|}{ Multivariate } \\
\hline & HR $(95 \% \mathrm{Cl})$ & $P$ & $\mathrm{HR}(95 \% \mathrm{Cl})$ & $P$ \\
\hline Age & 1.004 (0.985 to 1.023$)$ & 0.707 & 1.016 (0.996 to 1.037$)$ & 0.112 \\
\hline Gender & $1.105(0.753$ to 1.621$)$ & 0.611 & $1.050(0.712$ to 1.549$)$ & 0.806 \\
\hline TNM stage & $1.322(1.202$ to 1.454$)$ & $0.000^{*}$ & 1.305 (1.021 to 1.669$)$ & $0.034^{*}$ \\
\hline Tumor stage & 1.696 (1.348 to 2.134$)$ & $0.000^{*}$ & 1.173 (0.875 to 1.573$)$ & 0.286 \\
\hline Metastasis stage & 1.594 (0.871 to 2.918$)$ & 0.13 & 0.528 (0.181 to 1.537$)$ & 0.241 \\
\hline Node stage & $1.820(1.474$ to 2.247$)$ & $0.000^{*}$ & 1.180 (0.836 to 1.666$)$ & 0.345 \\
\hline NEIL3 & $1.760(1.456$ to 2.129$)$ & $0.000^{*}$ & 1.638 (1.338 to 2.005$)$ & $0.000^{*}$ \\
\hline
\end{tabular}

*Statistically significant

Subsequently, we analyzed mRNA sequencing data of TCGA cohort and acquired 370 genes co-expressed with NEIL3 (Additional file 3: Table S1, absolute Pearson correlation coefficient $>0.5, p<0.001)$. As showed in the protein-protein interaction network (PPI), there were 323 genes (yellow dots) positively related to NEIL3, versus 47 genes (blue dots) negatively related to NEIL3 (Fig. 5b). Next, we performed GO and KEGG enrichment analyses and displayed the top 30 items. The GO analysis indicated that the co-expressed genes were significantly enriched in nuclear cell cycle DNA replication, DNA strand elongation, and DNA replication initiation 
in the biological process group; condensed chromosome outer kinetochore and spindle midzone in the cellular component group; $3^{\prime}-5^{\prime}$ DNA helicase activity in the molecular function group (Fig. 5c). Similarly, the KEGG pathway enrichment analyses showed that these genes were mainly enriched in the cell cycle, oocyte meiosis and DNA replication (Fig. 5d). Taking 370 co-expressed genes into analysis via the Cytoscape software cytoHubba plugin, we filtered 10 hub genes according to node degree: TOP2A, CCNA2, BUB1B, CDC45, BUB1, CDK1,

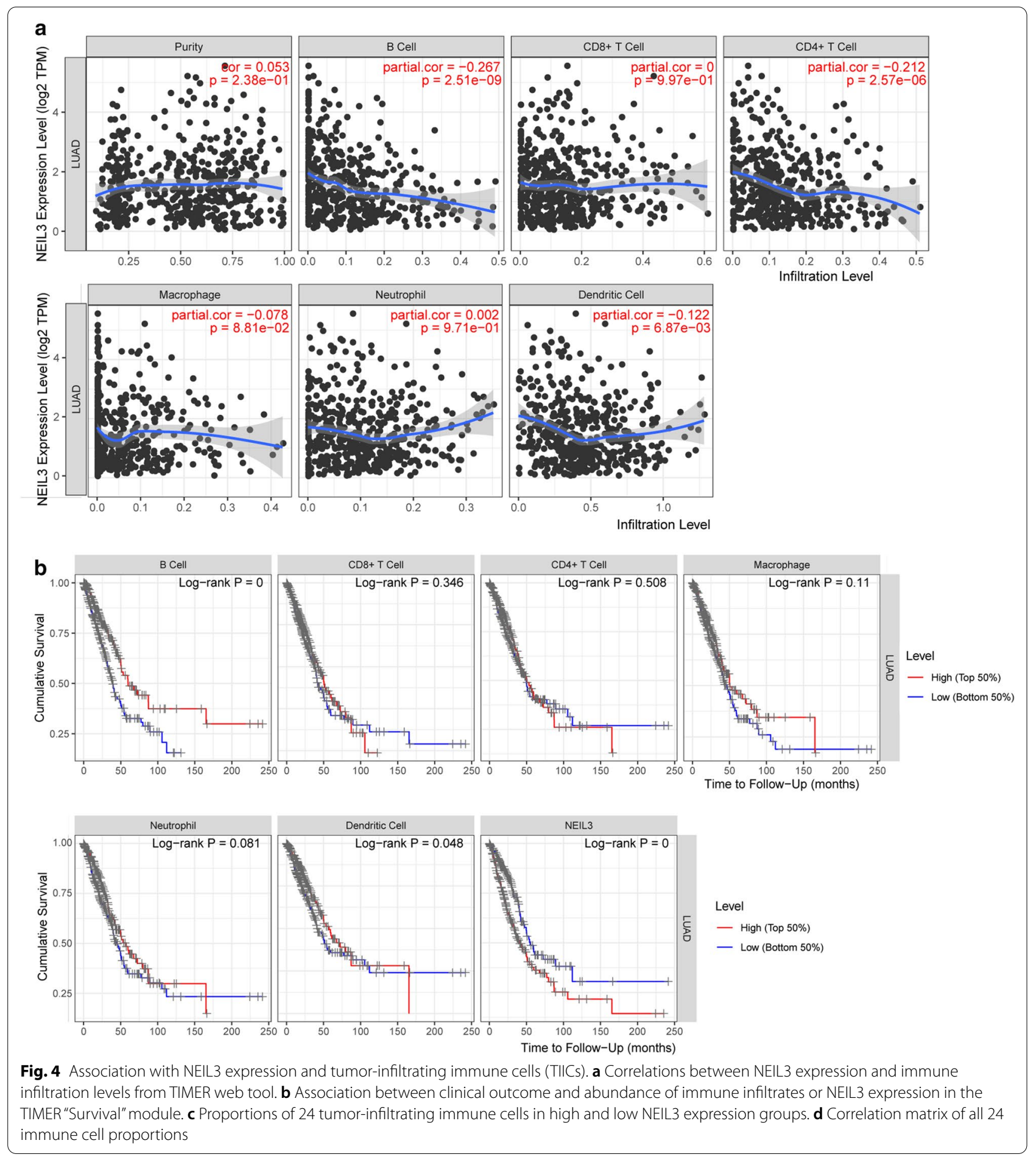



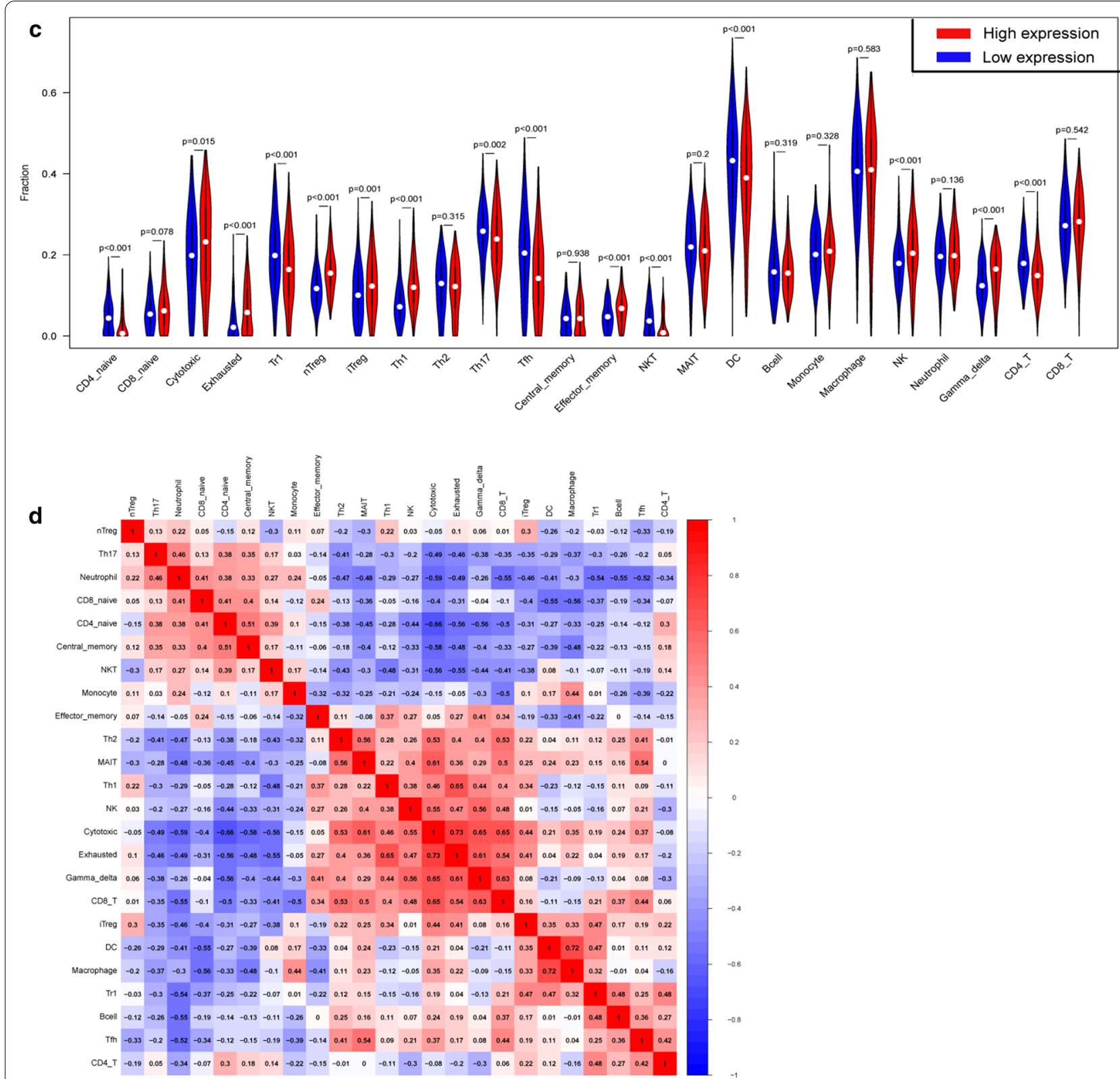

Fig. 4 continued

NCAPG, KIF23, UBE2C, and CCNB2 (Fig. 5e, Table 4). Several hub genes (CDK1, CCNA2, CDC45, and CCNB2) played a vital role in cell cycle progression, whereas genomic instability contributed to potentiate tumorigenesis [27].

\section{Establishment and evaluation of prognostic signature for LUAD patients}

Based on the TCGA LUAD cohorts, we verified that all 10 of hub genes were conspicuously overexpressed in LUAD tissues versus normal lung tissues $(p<0.05$; Additional file 1: Fig. S1). In addition, the high expressions of nine hub genes (all but TOP2A) were remarkably associated with poor prognosis in LUAD patients $(\mathrm{p}<0.05$; Additional file 2: Fig. S2). 
(See figure on next page.)

Fig. 5 Functional enrichment analysis and protein-protein interaction network of NEIL3 and genes co-expressed with NEIL3. a KEGG signaling pathway enrichment analysis of the high and low NEIL3 expression groups via GSEA software. $\mathbf{b}$ The PPI network among genes co-expressed with NEIL3. The yellow dots indicate a positive correlation with NEIL3, whereas the blue dots indicate a negative correlation. $\mathbf{c}$, $\mathbf{d}$ KEGG enrichment analysis and GO analysis of the co-expressed genes. e The 10 hub genes among the 370 co-expressed genes identified by the Cytoscape software CytoHubba plugin

Next, we attempted to establish a prognostic signature based on the expressions of NEIL3 and the other nine hub genes (Table 4). According to the median risk score (cutoff $=0.926)$, the LUAD patients were divided into two groups with discrete clinical outcomes for OS. Figure 6a shows the distribution of risk scores in the LUAD dataset. More deaths occurred in the high risk-score group than in the low risk score group (Fig. 6b). Meanwhile, the expressions of these 10 genes were up-regulated in the high-risk group (Fig. 6c). Figure 6d shows that patients in the low-risk group had significantly better OS than others in the Kaplan-Meier analysis $(p<0.05)$. Analysis of the association between risk core and various clinical features revealed that an increased risk score was correlated with more advanced stage, the larger tumor size and poor outcome (Fig. 6e, f, g).

The univariate and multivariate Cox analyses revealed that the risk score of a 10-gene signature was an independent prognostic factor for LUAD patients $(p<0.05$, Fig. 7a, b). After ROC analysis, we observed a marked predictive advantage of the risk-score signature (area under the curve $[\mathrm{AUC}]=0.679)$, stage $(\mathrm{AUC}=0.739)$ and lymph node metastasis $(\mathrm{AUC}=0.680$ ) (Fig. 7c).

Finally, we selected a test cohort based on GSE50081 (127 LUAD patients with TNM stage I \& II). In line with the TCGA cohort, the risk score of the 10-gene prognostic signature could be an independent prognostic factor for LUAD patients $(p<0.001$; Fig. $7 \mathrm{e}$, Table 5). Patients in the high risk score group had poor OS ( $p<0.001$; Fig. $6 \mathrm{~d})$, and the AUC values of the $1-, 3-$, and 5-year ROC curves were 0.676, 0.788, and 0.766 , respectively $(p<0.001$; Fig. $7 \mathrm{f})$. These evidences strongly suggests that the 10-gene prognostic signature has superior diagnostic accuracy and may benefit LUAD patients in the early stage.

\section{Discussion}

LUAD, the most common type of malignant tumors, has significant morbidity and mortality rates [28]. Growing scientific research has focused on searching for effective treatment methods and sensitive biomarkers to improve the 5-year survival rate and life quality of LUAD patients. NEIL3, a DNA glycosylase of the BER pathway, repairs telomere oxidative damage and protects telomere integrity in cells with a high proliferative capacity during the $S$ phase, which may explain the advanced Tumor stage in NEIL3 overexpressing patients [17]. In addition, some studies indicated NEIL3 as a cell cycle dependent gene was regulated by BRG1 in breast cancer cells and that NEIL3 overexpression may facilitate distant metastasis in primary melanoma $[15,19]$. Tran et al. found NEIL3 was overexpressed in a variety of tumors such as pancreatic adenocarcinoma, lower grade glioma, and kidney papillary cell carcinoma; furthermore, NEIL3 overexpressed tumors accumulate mutation and chromosomal variations [29]. As mentioned in the literature review, NEIL3 plays a crucial role in preventing autoimmunity and cell proliferation [11, 13]. Here a comprehensive bioinformatics analysis was performed based on gene transcript profiles of LUAD from TCGA and GEO databases. Combined with the IHC-scores of our 406 patients cohort, NEIL3 expression was up-regulated in LUAD tissues and correlated with clinicopathological characteristics, especially advanced TNM stage and large tumor size. Meanwhile, the Cox regression analysis results demonstrated that NEIL3 may serve as an independent prognostic predictor in LUAD patients.

Over the past decade, immunotherapy has been a well-known promising cancer treatment with amazing achievements in treating various refractory malignancies. The pivotal strategy of immunotherapy is to interfere with immune checkpoints expressed in immune cells [30]. Immune cells are a crucial part of the immune microenvironment, including tumor infiltrating lymphocytes (TILs), tumor-associated macrophages (TAM), dendritic cells (DC), and myeloid-derived suppressor cells (MDSCs) [31, 32]. As reported in the immunoediting theory, tumor invasive immune cells (TIICs) play a "double-edged sword" role in the development of cancers. A large number of studies have found the occurrence and development of lung adenocarcinoma not only depends on the lung cancer cells themselves but also is regulated by the tumor-infiltrating immune cells in the lung cancer microenvironment [33].

Based on the TIMER database review, NEIL3 expression had a significant negative correlation with $B$ cells, CD4 ${ }^{+} \mathrm{T}$ cells, and DCs. The ImmuCellAI analysis revealed nTreg, iTreg, and Exhausted $\mathrm{T}$ cells were increased in the high NEIL3 expression group, whereas Th17 cells, DCs and CD4 ${ }^{+} \mathrm{T}$ cells were decreased. Th17 cells play a contradictory role in tumorigenesis and might be associated with secreting cytokines such as IL-17A, 


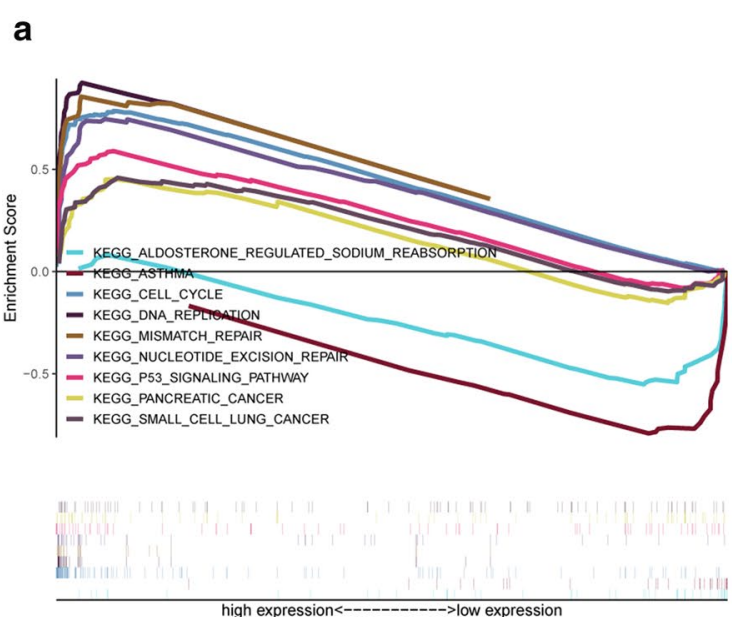

C

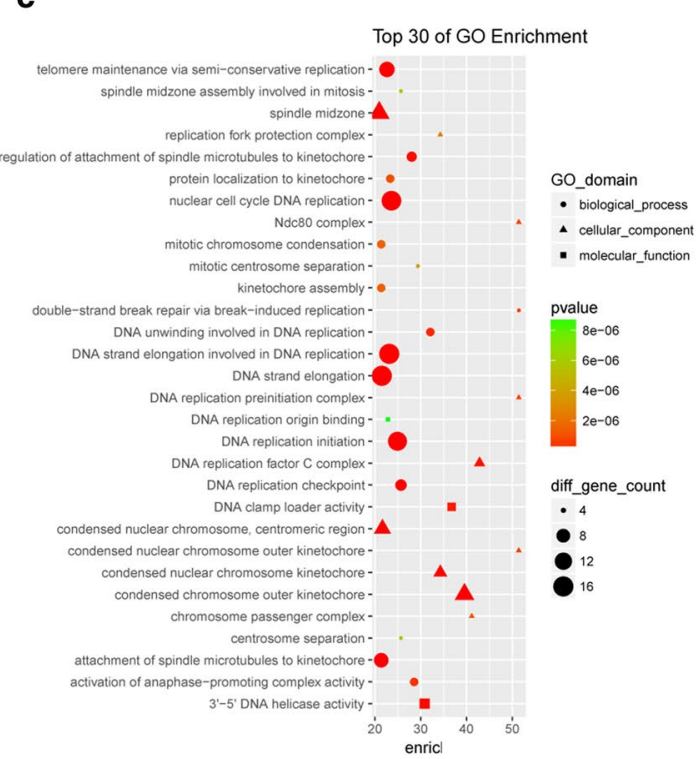

b

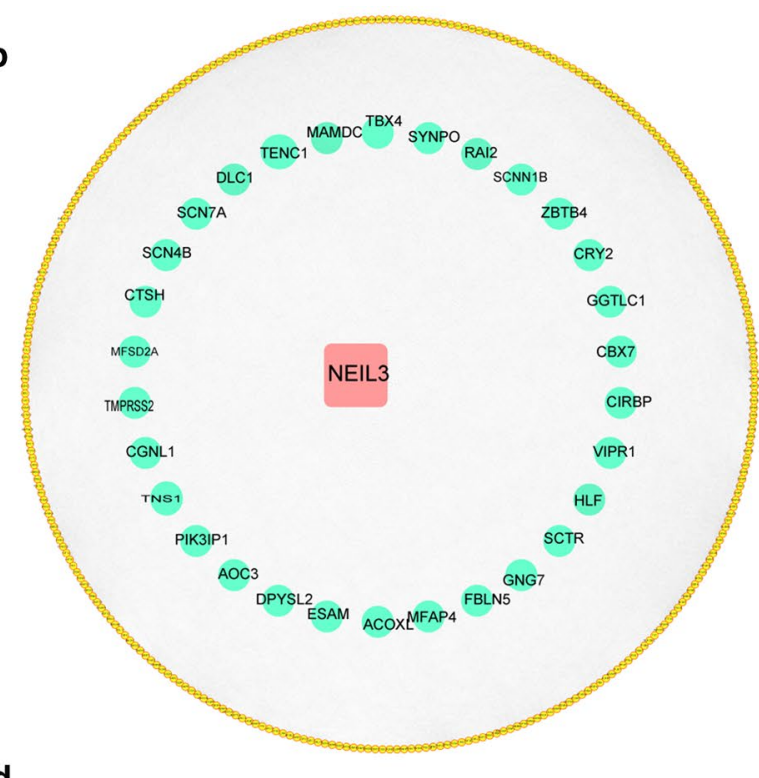

d

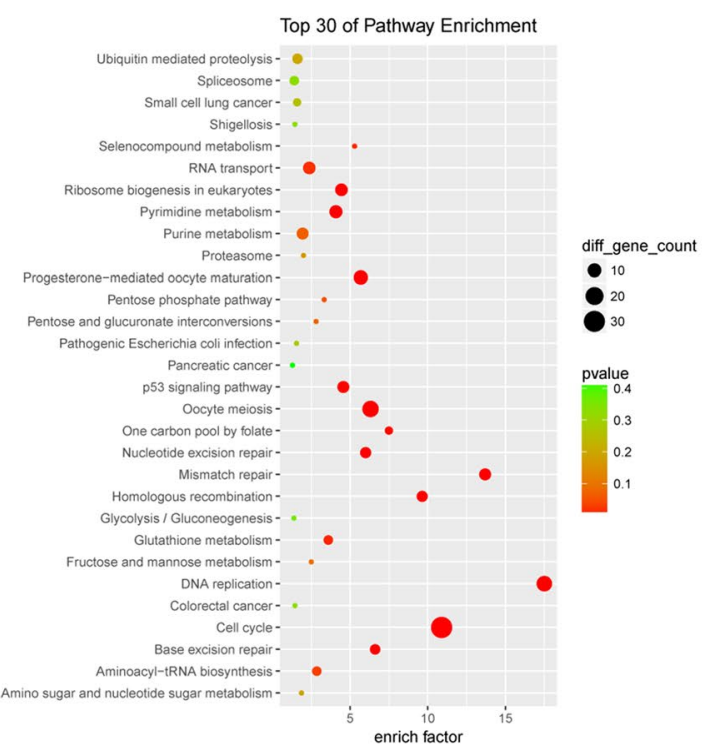

e

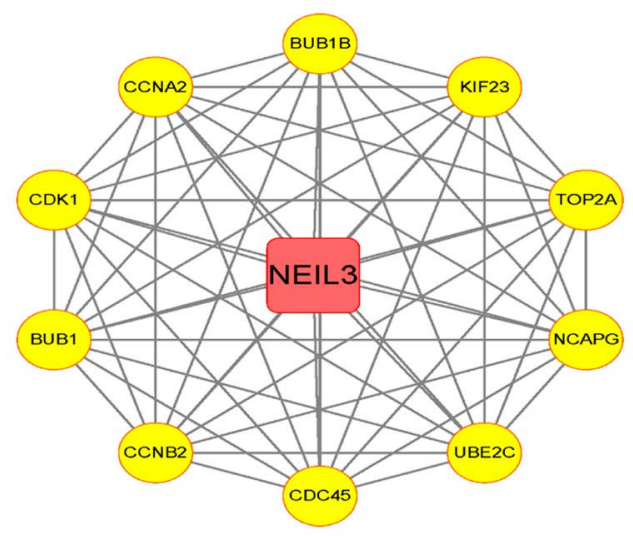


Table 4 The hub genes co-expressed with NEIL3

\begin{tabular}{llll}
\hline Gene & Cor & Pvalue & Group \\
\hline TOP2A & 0.635 & $1.52 \mathrm{E}-63$ & Positive \\
CCNA2 & 0.726 & $2.36 \mathrm{E}-91$ & Positive \\
BUB1B & 0.683 & $4.49 \mathrm{E}-77$ & Positive \\
CDC45 & 0.631 & $2.04 \mathrm{E}-62$ & Positive \\
BUB1 & 0.669 & $7.54 \mathrm{E}-73$ & Positive \\
CDK1 & 0.668 & $1.34 \mathrm{E}-72$ & Positive \\
NCAPG & 0.7 & $2.21 \mathrm{E}-82$ & Positive \\
KIF23 & 0.672 & $1.49 \mathrm{E}-73$ & Positive \\
UBE2C & 0.591 & $3.82 \mathrm{E}-53$ & Positive \\
CCNB2 & 0.674 & $3.35 \mathrm{E}-74$ & Positive \\
\hline
\end{tabular}

IL-17F, IL-21, and IFN- $\gamma+$ Th17 cells as well as effector lymphocytes, including Th1, Tc1, and NK cells [34-36]. Treg cells could inhibit the activation of $\mathrm{T}$ lymphocytes by secreting cytokines such as IL-4, IL-10, and TGF- $\beta$, which regulate the immune function of tumor patients and promote the proliferation of lung cancer cells [37]. Evidence indicates that DCs induced antitumor immunity and inhibit the formation of new blood vessels in tumors in the lung cancer microenvironment [38]. These findings suggest that NEIL3 overexpression could increase the proportion of $\mathrm{T}$ regulatory cells and inhibit the antitumor function of Th17 cells and DCs, which predicted a poor
OS and advanced TNM stage. Together this evidence demonstrates that NEIL3 plays a pivotal role in the regulation of immune infiltrating cells and could be considered a novel immune-related therapeutic target in LUAD. The molecular mechanism underlying NEIL3 gene and tumor microenvironment is still not known, which is our limitation and next crucial research question.

Equally important, GSEA analysis showed that the cell cycle and P53 signaling pathway were enriched in cases of high NEIL3 expression. As we all know, cell cycle proteins dysregulations is related to uncontrolled proliferation of a malignant tumor and becomes an attractive target in cancer therapy [39]. This work selected nine prognosisassociated hub genes among 370 genes co-expressed with NEIL3: CCNA2, BUB1B, CDC45, BUB1, CDK1, NCAPG, KIF23, UBE2C and CCNB2 (Table 3, Additional file 2: Fig. S2). CDK1, a cyclin-dependent kinase, plays an important part in regulating cell cycle progression and reportedly increases cellular proliferation in various cancers if dysregulated [40]. CCNA2 and CCNB2 are cyclin family proteins. CCNA2 was recognized as an effective prognostic marker in prostate, colon, lung, and liver cancers $[41,42]$. In line with CCNA2, CCNB2 increased the risk of multiple cancer prognoses such as such as adrenocortical carcinoma [43], lung cancer [44], breast cancer [45], and colorectal adenocarcinoma [46].

Our result showed that NEIL3 and nine hub genes were in a tight co-expressed relationship. Combining
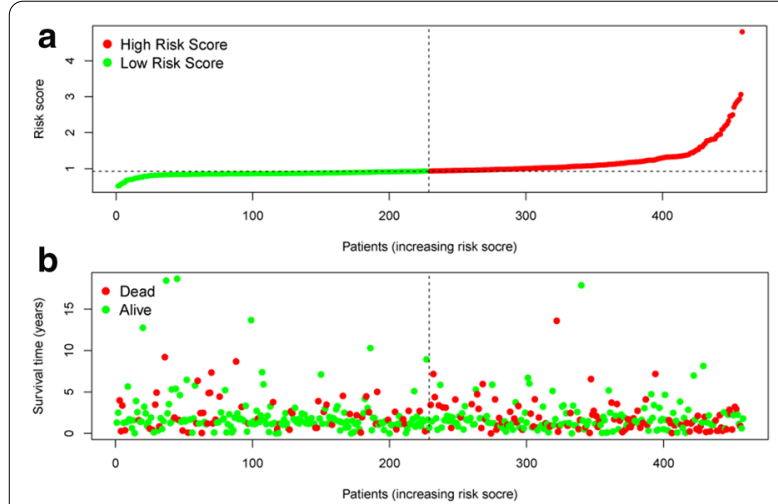

C

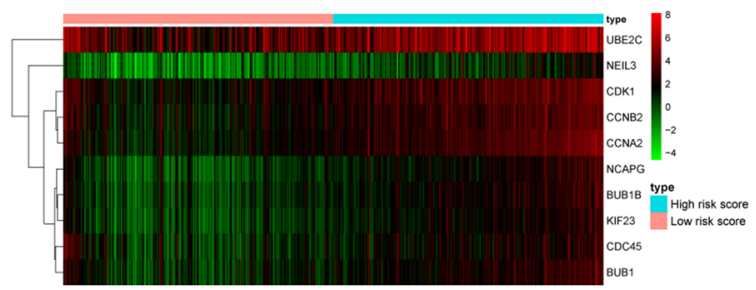

d

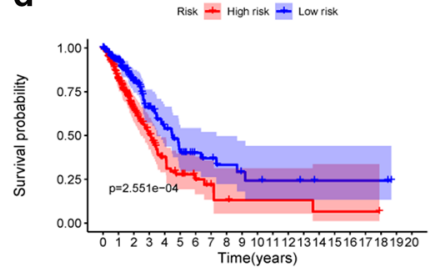

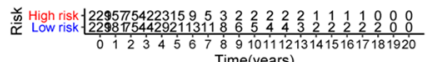

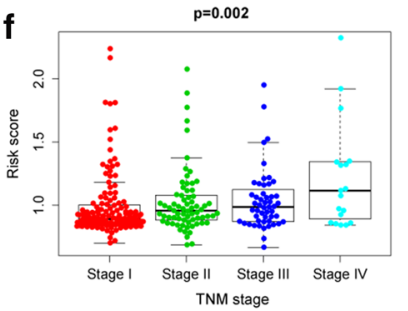

e
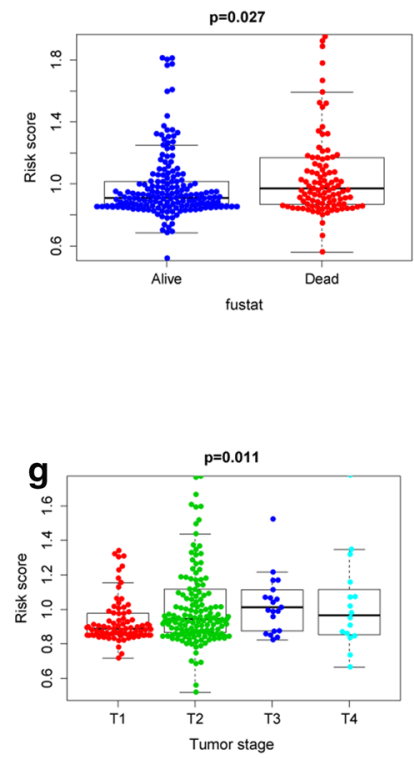

Fig. 6 Relation between signature and cancer risk. a Distribution of risk score. b Proportion of deaths was increased in the high risk score group. c Hierarchical clustering of the 10 genes between the low and high risk groups. Red, up-regulated; green, down-regulated. $\mathbf{d}-\mathbf{g} \mathrm{A}$ higher risk score was remarkably associated with shorter overall survival, poorer clinical outcomes, more advanced TNM stage, and larger tumor size $(p<0.05)$ 


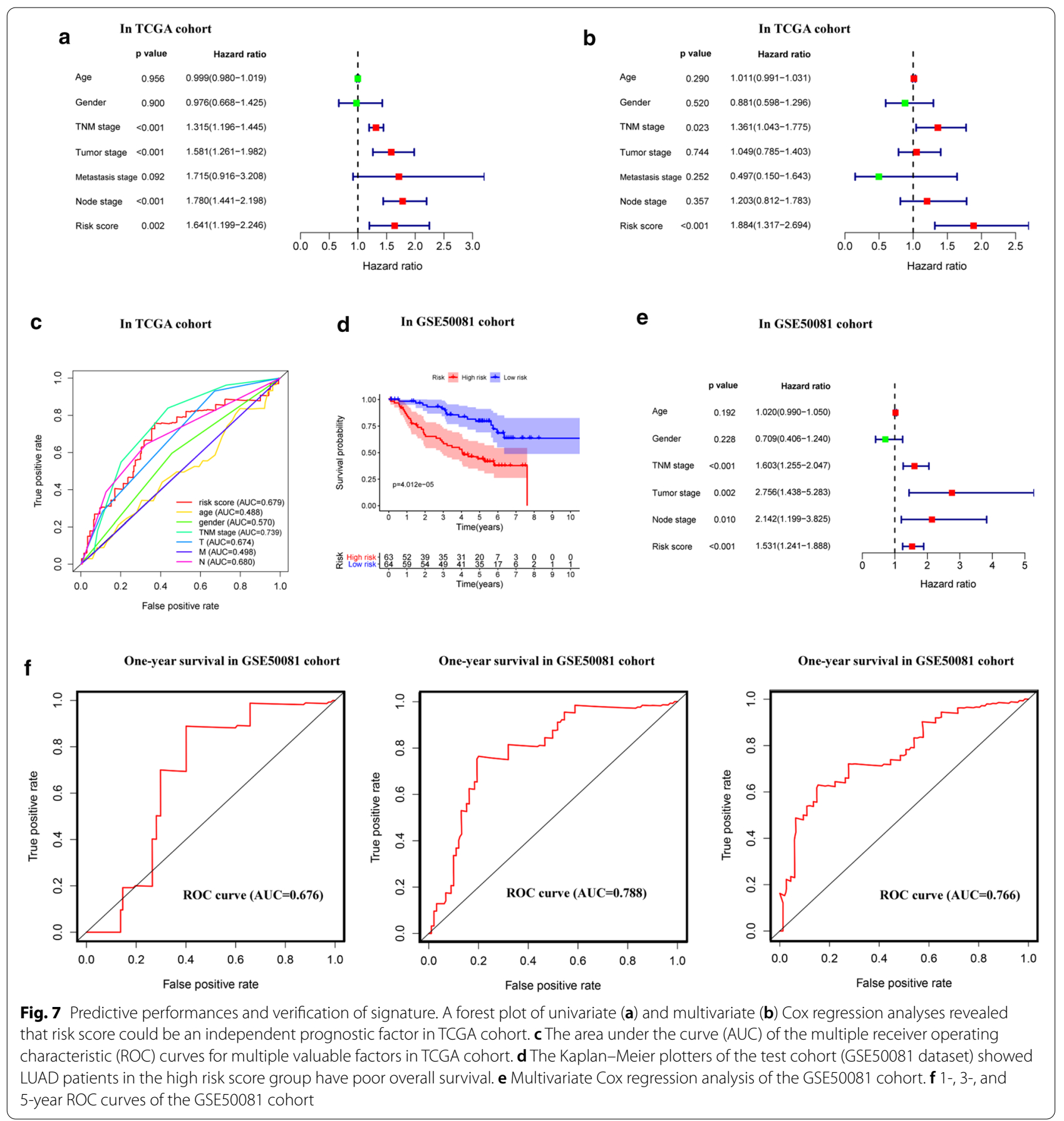

the expression levels of the nine hub genes and NEIL3, a 10-gene prognostic signature was constructed to accurately predict the prognostic risk of LUAD patients. Multivariate Cox regression analyses proved that the risk score was an independent prognostic factor correlated with TNM stage and tumor size. Analysis of the test cohort data achieved the same results. As early as 2007, Chen et al. attempted to develop gene signatures correlated with NSCLC clinical outcomes and finally developed a five-gene prognostic signature for NSCLC [47]. Since then, scholars have performed a great number of studies to explore novel gene signature as effective classifier to predict prognosis. Sun et al. [48] identified a immune-related prognostic signature, but the AUC of verification set GSE50081 was only about 0.617, and Wang et al. [49] identified a 4-gene signature, but the 
Table 5 Univariate and multivariate analyses of factors in the GSE50081 cohort using Cox regression

\begin{tabular}{|c|c|c|c|c|}
\hline \multirow[t]{2}{*}{ Variable } & \multicolumn{2}{|l|}{ Univariate } & \multicolumn{2}{|l|}{ Multivariate } \\
\hline & $\mathrm{HR}(95 \% \mathrm{Cl})$ & $P$ & $\mathrm{HR}(95 \% \mathrm{Cl})$ & $P$ \\
\hline Age & $1.020(0.990$ to 1.050$)$ & 0.192 & 1.009 (0.978 to 1.041$)$ & 0.562 \\
\hline Gender & $0.709(0.406$ to 1.240$)$ & 0.228 & 0.701 (0.395 to 1.244$)$ & 0.225 \\
\hline TNM stage & 1.603 (1.255 to 2.047$)$ & $0.000^{*}$ & 0.904 (0.126 to 6.476) & 0.92 \\
\hline Tumor stage & 2.756 (1.438 to 5.283) & $0.002^{*}$ & $2.229(0.204$ to 24.396$)$ & 0.512 \\
\hline Node stage & 2.142 (1.199 to 3.825$)$ & $0.010^{*}$ & 2.940 (0.056 to 155.468$)$ & 0.594 \\
\hline Risk score & $1.531(1.241$ to 1.888$)$ & $0.000^{*}$ & 1.577 (1.203 to 2.067$)$ & $0.001^{*}$ \\
\hline
\end{tabular}

*Statistically significant

average AUC was less than 0.7. In addition, Liu et al. [50] identified a 22-gene signature through analysis of autophagy gene expression. Although the AUC is high, the large number of genes that needs to be detected makes this analysis impractical for clinical use. By contrast, our signature has a high AUC using 10 genes, which makes it conducive to clinical application and provides a theoretical basis for the development of new targeted therapies.

NEIL3 expression and the 10-gene signature were proven to be independent predictors for LUAD patients using bioinformatics technology in this study. However, identifying NEIL3 in LUAD cell lines, exploring the underlying tumorigenesis mechanism, and designing novel potent selective NEIL3-targeted drugs require further research.

\section{Conclusion}

In summary, this study revealed that NEIL3 was overexpressed in LUAD and identified it as a new diagnostic biomarker for LUAD patients. Increased NEIL3 expression was related to advanced stage and larger tumor size as an independent diagnostic factor of poor prognosis in LUAD patients. Moreover, NEIL3 may play an important role in regulating immune-infiltrating cells such as regulatory $\mathrm{T}$ cells and DCs and may affect LUAD cell proliferations. The cell cycle and P53 signaling pathway is the major pathway affected by NEIL3 in LUAD. And finally, we constructed a 10-gene prognostic signature (NEIL3 and nine co-expressed hub genes) to accurately predict prognostic risk of LUAD patients. The results based on the test cohort proved that the 10-gene signature had precise diagnostic accuracy. Meanwhile, NEIL3 could be a promising biomarker for diagnosis and treatment and correlates with immune infiltration in LUAD.

\section{Abbreviations}

LUAD: Lung adenocarcinoma; NEIL3: DNA endonuclease VIII-like 3; GEO: Gene expression omnibus; TCGA: The cancer genome atlas; RT-qPCR: Real-time quantitative PCR; GO: Gene ontology; KEGG: Kyoto encyclopedia of genes and genomes; PPI: Protein-protein interaction; OS: Overall survival; RFS: Relapsefree survival; IHC-score: Immunohistochemistry score; TIICs: Tumor-infiltrating immune cells; ImmuCellAI: Immune Cell Abundance Identifier; TIMER: Tumor Immune Estimation Resource; nTreg: T regulatory cells; iTreg: Induced T regulatory cells; AUC: The area under the curve; Cl: Confidence interval.

\section{Supplementary Information}

The online version contains supplementary material available at https://doi. org/10.1186/s12935-021-01938-4.

Additional file 1: Figure S1. The expression levels of the 10 hub genes in LUAD tissues and normal lung tissues.

Additional file 2: Figure S2. The overall survival Kaplan-Meier plotters of the 10 hub genes in TCGA LUAD cohort.

Additional file 3: Table S1. The 370 significant co-expressed genes.

\section{Acknowledgements}

We thank Dr. Zhou for useful suggestions, Dr. Yang and Dr. Liu for effective direction during writing.

\section{Authors' contributions}

$C Z$, and $J L$ designed the current study and wrote the manuscript; $H M Z, X Q$, and $\mathrm{HS}$ contributed to the statistical analysis process and interpretation of data; XWC, MSZ, TTB, and LL collected and used new software in the work; JGZ and YFL acquired funding and substantively revised the work. All authors read and approved the final manuscript.

\section{Funding}

This study was funded by grants from Jiangsu Post-doctoral Foundation Research Project, China (No. 2019Z142), Key Talents of Medical Science in Jiangsu Province, China (No. QNRC2016682), and Key Scientific and Technological Projects in Nantong City, Jiangsu, China (MS22019015).

\section{Availability of data and materials}

The datasets of this study can be found in the Gene Expression Omnibus (GEO) repository (https://www.ncbi.n/m.nih.gov/gds/) and The Cancer Genome Atlas (https://www.ncbi.nlm.nih.gov/gds/).

\section{Declarations}

\section{Ethics approval and consent to participate}

The studies were approved and consented to by the Ethics Committee of the Affiliated Hospital of Nantong University. The patients provided their written informed consent to participate in this research.

\section{Consent for publication}

Not applicable.

\section{Competing interests}

The authors declare that they have no competing interests.

\section{Author details}

${ }^{1}$ Nantong University, Nantong 226001, China. ${ }^{2}$ Department of Pathology, Affiliated Hospital of Nantong University, Nantong 226001, China. ${ }^{3}$ Department of Chemotherapy, Affiliated Hospital of Nantong University, Nantong 226001, China. ${ }^{4}$ Department of Orthopedics, Second People's Hospital of Jingmen, Jingmen 448000, China.

Received: 20 October 2020 Accepted: 13 April 2021

Published online: 20 April 2021 


\section{References}

1. Siegel RL, Miller KD, Jemal A. Cancer statistics, 2020. CA Cancer J Clin. 2020;70(1):7-30.

2. Yang L, Li L, Zhou Z, Liu Y, Sun J, Zhang X, Pan H, Liu S. SP1 induced long non-coding RNA LINC00958 overexpression facilitate cell proliferation, migration and invasion in lung adenocarcinoma via mediating miR625-5p/CPSF7 axis. Cancer Cell Int. 2020;20:24.

3. Zeng X, Liu Q, Yang Y, Jia W, Li S, He D, Ma R. Placenta-specific protein 8 promotes the proliferation of lung adenocarcinoma PC-9 cells and their tolerance to an epidermal growth factor receptor tyrosine kinase inhibitor by activating the ERK signaling pathway. Oncol Lett. 2019:18(5):5621-7.

4. Li P, Guo P, Lin C, He M, Zhu X, Liu C, Tang J, Wang W, Liang W. The synergistic effect of propofol and ulinastatin suppressed the viability of the human lung adenocarcinoma epithelial A549 cell line. Oncol Lett. 2018;16(4):5191-9.

5. Luo C, Lei M, Zhang Y, Zhang Q, Li L, Lian J, Liu S, Wang L, Pi G, Zhang Y. Systematic construction and validation of an immune prognostic model for lung adenocarcinoma. J Cell Mol Med. 2020;24(2):1233-44.

6. Wang Q, Li M, Yang M, Yang Y, Song F, Zhang W, Li X, Chen K. Analysis of immune-related signatures of lung adenocarcinoma identified two distinct subtypes: implications for immune checkpoint blockade therapy. Aging. 2020;12(4):3312-39.

7. Liu M, Bandaru V, Bond JP, Jaruga P, Zhao X, Christov PP, Burrows CJ, Rizzo CJ, Dizdaroglu M, Wallace SS. The mouse ortholog of NEIL3 is a functional DNA glycosylase in vitro and in vivo. Proc Natl Acad Sci USA. 2010;107(11):4925-30.

8. Morland I, Rolseth V, Luna L, Rognes T, Bjoras M, Seeberg E. Human DNA glycosylases of the bacterial Fpg/MutM superfamily: an alternative pathway for the repair of 8-oxoguanine and other oxidation products in DNA. Nucleic Acids Res. 2002;30(22):4926-36.

9. Krokeide SZ, Laerdahl JK, Salah M, Luna L, Cederkvist FH, Fleming AM, Burrows CJ, Dalhus B, Bjoras M. Human NEIL3 is mainly a monofunctional DNA glycosylase removing spiroimindiohydantoin and guanidinohydantoin. DNA Repair. 2013;12(12):1159-64.

10. Zhou J, Chan J, Lambele M, Yusufzai T, Stumpff J, Opresko PL, Thali M, Wallace SS. NEIL3 repairs telomere damage during $S$ phase to secure chromosome segregation at mitosis. Cell Rep. 2017;20(9):2044-56.

11. Massaad MJ, Zhou J, Tsuchimoto D, Chou J, Jabara H, Janssen E, Glauzy S, Olson BG, Morbach H, Ohsumi TK, et al. Deficiency of base excision repair enzyme NEIL3 drives increased predisposition to autoimmunity. J Clin Invest. 2016:126(11):4219-36.

12. Jalland CM, Scheffler K, Benestad SL, Moldal T, Ersdal C, Gunnes G, Suganthan R, Bjoras M, Tranulis MA. Neil3 induced neurogenesis protects against prion disease during the clinical phase. Sci Rep. 2016;6:37844.

13. Regnell CE, Hildrestrand GA, Sejersted Y, Medin T, Moldestad O, Rolseth V, Krokeide SZ, Suganthan R, Luna L, Bjoras M, et al. Hippocampal adult neurogenesis is maintained by Neil3-dependent repair of oxidative DNA lesions in neural progenitor cells. Cell Rep. 2012;2(3):503-10.

14. Hildrestrand GA, Neurauter CG, Diep DB, Castellanos CG, Krauss S, Bjoras M, Luna L. Expression patterns of Neil3 during embryonic brain development and neoplasia. BMC Neurosci. 2009;10:45.

15. Sobczak M, Pietrzak J, Ploszaj T, Robaszkiewicz A. BRG1 activates proliferation and transcription of cell cycle-dependent genes in breast cancer cells. Cancers. 2020;12(2):349.

16. Sobczak M, Pitt AR, Spickett CM, Robaszkiewicz A. PARP1 co-regulates EP300-BRG1-dependent transcription of genes involved in breast cancer cell proliferation and DNA repair. Cancers. 2019;11(10):1539.

17. Kauffmann A, Rosselli F, Lazar V, Winnepenninckx V, Mansuet-Lupo A, Dessen $\mathrm{P}$, van den Oord JJ, Spatz A, Sarasin A. High expression of DNA repair pathways is associated with metastasis in melanoma patients. Oncogene. 2008:27(5):565-73.

18. de Sousa JF, Torrieri R, Serafim RB, Di Cristofaro LF, Escanfella FD, Ribeiro R, Zanette DL, Paco-Larson ML, da Silva WA Jr, Tirapelli DP, et al. Expression signatures of DNA repair genes correlate with survival prognosis of astrocytoma patients. Tumour Biol. 2017:39(4):1010428317694552.

19. Sousa JF, Serafim RB, Freitas LM, Fontana CR, Valente V. DNA repair genes in astrocytoma tumorigenesis, progression and therapy resistance. Genet Mol Biol. 2019;43(1 suppl 1):e20190066.

20. Mo XG, Liu W, Yang Y, Imani S, Lu S, Dan G, Nie X, Yan J, Zhan R, Li X, et al. NCF2, MYO1F, S1PR4, and FCN1 as potential noninvasive diagnostic biomarkers in patients with obstructive coronary artery: a weighted gene co-expression network analysis. J Cell Biochem. 2019;120(10):18219-35.

21. Lin QG, Liu W, Mo YZ, Han J, Guo ZX, Zheng W, Wang JW, Zou XB, Li AH, Han F. Development of prognostic index based on autophagy-related genes analysis in breast cancer. Aging. 2020;12(2):1366-76.

22. Li T, Fan J, Wang B, Traugh N, Chen Q, Liu JS, Li B, Liu XS. TIMER: a web server for comprehensive analysis of tumor-infiltrating immune cells. Cancer Res. 2017;77(21):e108-10.

23. Miao YR, Zhang Q, Lei Q, Luo M, Xie GY, Wang H, Guo AY. ImmuCellAl: a unique method for comprehensive T-cell subsets abundance prediction and its application in cancer immunotherapy. Adv Sci. 2020;7(7):1902880.

24. Yin F, Yi S, Wei L, Zhao B, Li J, Cai X, Dong C, Liu X. Microarray-based identification of genes associated with prognosis and drug resistance in ovarian cancer. J Cell Biochem. 2019;120(4):6057-70.

25. Li B, Severson E, Pignon JC, Zhao H, Li T, Novak J, Jiang P, Shen H, Aster JC, Rodig S, et al. Comprehensive analyses of tumor immunity: implications for cancer immunotherapy. Genome Biol. 2016;17(1):174.

26. Lohr M, Edlund K, Botling J, Hammad S, Hellwig B, Othman A, Berglund A, Lambe M, Holmberg L, Ekman S, et al. The prognostic relevance of tumour-infiltrating plasma cells and immunoglobulin kappa C indicates an important role of the humoral immune response in non-small cell lung cancer. Cancer Lett. 2013;333(2):222-8.

27. Thu KL, Soria-Bretones I, Mak TW, Cescon DW. Targeting the cell cycle in breast cancer: towards the next phase. Cell Cycle. 2018;17(15):1871-85.

28. Torre LA, Siegel RL, Jemal A. Lung cancer statistics. Adv Exp Med Biol. 2016;893:1-19.

29. Tran OT, Tadesse S, Chu C, Kidane D. Overexpression of NEIL3 associated with altered genome and poor survival in selected types of human cancer. Tumour Biol. 2020;42(5):1010428320918404.

30. Han B, Sun Y, Yang D, Zhang H, Mo S, Chen X, Lu H, Mao X, Hu J. USP22 promotes development of lung adenocarcinoma through ubiquitination and immunosuppression. Aging. 2020;12:6990.

31. Klein-Goldberg A, Maman S, Witz IP. The role played by the microenvironment in site-specific metastasis. Cancer Lett. 2014;352(1):54-8.

32. Merlo A, Dalla Santa S, Dolcetti R, Zanovello P, Rosato A. Reverse immunoediting: when immunity is edited by antigen. Immunol Lett. 2016;175:16-20.

33. Zheng $X, H u Y$, Yao $C$. The paradoxical role of tumor-infiltrating immune cells in lung cancer. Intractable Rare Dis Res. 2017;6(4):234-41.

34. Nalbant A, Eskier D. Genes associated with T helper 17 cell differentiation and function. Front Biosci. 2016;8:427-35.

35. Duan MC, Han W, Jin PW, Wei YP, Wei Q, Zhang LM, Li JC. Disturbed Th17/ Treg balance in patients with non-small cell lung cancer. Inflammation. 2015;38(6):2156-65.

36. Jones SA, Sutton CE, Cua D, Mills KH. Therapeutic potential of targeting IL-17. Nat Immunol. 2012;13(11):1022-5.

37. Marshall EA, Ng KW, Kung SH, Conway EM, Martinez VD, Halvorsen EC, Rowbotham DA, Vucic EA, Plumb AW, Becker-Santos DD, et al. Emerging roles of T helper 17 and regulatory $T$ cells in lung cancer progression and metastasis. Mol Cancer. 2016;15(1):67.

38. Palucka K, Banchereau J. Cancer immunotherapy via dendritic cells. Nat Rev Cancer. 2012;12(4):265-77.

39. Otto T, Sicinski P. Cell cycle proteins as promising targets in cancer therapy. Nat Rev Cancer. 2017;17(2):93-115.

40. Izadi S, Nikkhoo A, Hojjat-Farsangi M, Namdar A, Azizi G, Mohammadi $H$, Yousefi M, Jadidi-Niaragh F. CDK1 in breast cancer: implications for theranostic potential. Anticancer Agents Med Chem. 2020;20(7):758-67.

41. Kim DH, Park SE, Kim M, Ji Yl, Kang MY, Jung EH, Ko E, Kim Y, Kim S, Shim $Y M$, et al. A functional single nucleotide polymorphism at the promoter region of cyclin $\mathrm{A} 2$ is associated with increased risk of colon, liver, and lung cancers. Cancer. 2011;117(17):4080-91.

42. Yang R, Du Y, Wang L, Chen Z, Liu X. Weighted gene co-expression network analysis identifies CCNA2 as a treatment target of prostate cancer through inhibiting cell cycle. J Cancer. 2020;11(5):1203-11.

43. Fernandez-Ranvier GG, Weng J, Yeh RF, Khanafshar E, Suh I, Barker C, Duh QY, Clark OH, Kebebew E. Identification of biomarkers of adrenocortical carcinoma using genomewide gene expression profiling. Arch Surg. 2008;143(9):841-6 (discussion 846)

44. Qian X, Song X, He Y, Yang Z, Sun T, Wang J, Zhu G, Xing W, You C. CCNB2 overexpression is a poor prognostic biomarker in Chinese NSCLC patients. Biomed Pharmacother. 2015;74:222-7. 
45. Shubbar E, Kovacs A, Hajizadeh S, Parris TZ, Nemes S, Gunnarsdottir K, Einbeigi Z, Karlsson P, Helou K. Elevated cyclin B2 expression in invasive breast carcinoma is associated with unfavorable clinical outcome. BMC Cancer. 2013;13:1.

46. Park SH, Yu GR, Kim WH, Moon WS, Kim JH, Kim DG. NF-Y-dependent cyclin B2 expression in colorectal adenocarcinoma. Clin Cancer Res. 2007;13(3):858-67.

47. Chen H-Y, Yu S-L, Chen C-H, Chang G-C, Chen C-Y, Yuan A, Cheng C-L, Wang $\mathrm{C}-\mathrm{H}$, Terng $\mathrm{H}-\mathrm{J}$, Kao S-F, et al. A five-gene signature and clinical outcome in non-small-cell lung cancer. N Engl J Med. 2007;356(1):11-20.

48. Sun S, Guo W, Wang Z, Wang X, Zhang G, Zhang H, Li R, Gao Y, Qiu B, Tan $F$, et al. Development and validation of an immune-related prognostic signature in lung adenocarcinoma. Cancer Med. 2020;9(16):5960-75.
49. Wang Y, Zhang Q, Gao Z, Xin S, Zhao Y, Zhang K, Shi R, Bao X. A novel 4-gene signature for overall survival prediction in lung adenocarcinoma patients with lymph node metastasis. Cancer Cell Int. 2019;19:100.

50. Liu Y, Wu L, Ao H, Zhao M, Leng X, Liu M, Ma J, Zhu J. Prognostic implications of autophagy-associated gene signatures in non-small cell lung cancer. Aging. 2019;11(23):11440-62.

\section{Publisher's Note}

Springer Nature remains neutral with regard to jurisdictional claims in published maps and institutional affiliations.
Ready to submit your research? Choose BMC and benefit from:

- fast, convenient online submission

- thorough peer review by experienced researchers in your field

- rapid publication on acceptance

- support for research data, including large and complex data types

- gold Open Access which fosters wider collaboration and increased citations

- maximum visibility for your research: over 100M website views per year

At BMC, research is always in progress.

Learn more biomedcentral.com/submissions 